\title{
Jogos Digitais no desenvolvimento de conceitos matemáticos sob perspectiva BYOD e abordagem m-Learning na escola pública
}

\author{
Adalberto Bosco Castro Pereira ${ }^{1}$, Flávio Soares Correa Da Silva ${ }^{1}$, Stela Conceição \\ Bertholo Piconez ${ }^{2}$, Josete Maria Zimmer² \\ ${ }^{1}$ Dept. of Computer Science IME-USP São Paulo, Brazil \\ ${ }^{2}$ Dept. of Education FE-USPSão Paulo, Brazil \\ \{bosco,fcs\}@ime.usp.br, spiconez@usp.br, josete.zimmer@gmail.com
}

\begin{abstract}
This paper presents the possibilities of using digital games for learning mathematical concepts. It uses a composition of mobile devices from the "Bring your own device" (BYOD) perspective and supported by social networking, in state elementary school II. Challenges were highlighted, such as absence of teacher training about the subject; problems with incompatible technology infrastructure with the appropriate educational activities and the need to develop a methodology about using digital games as a support in the construction of mathematical concepts.
\end{abstract}

Resumo. Este artigo apresenta as possibilidades de uso de jogos digitais para a aprendizagem de conceitos matemáticos. Utiliza uma composição de dispositivo móvel sob a perspectiva "Bring your own device" (BYOD) e apoio de redes sociais, em escola estadual de ensino fundamental II. Foram evidenciados desafios, tais como, a ausência de formação de professores sobre o tema; problemas com infraestrutura tecnológica incompativel com as atividades pedagógicas adequadas e a necessidade da criação de uma metodologia sobre o uso de jogos digitais como apoio na construção de conceitos matemáticos.

\section{Introdução}

É crescente o número de artigos internacionais de professores interessados em integrar jogos digitais de computador com conteúdos curriculares da escola [Roblyer 2006], [Furio et. al 2013]. Estes estudos demonstraram que os jogos digitais representam estratégia efetiva para complementar a construção de conhecimentos dos alunos [Wang e Chen 2010]. Comprovaram por meio deles que a integração tecnológica por meio dos games favorece interações intuitivas e positivas entre os alunos, auxiliando-os no engajamento ativo de suas atividades [Ardito et. al 2013].

Entretanto, o sistema educacional brasileiro ainda não conseguiu introduzir os jogos digitais ao currículo escolar. As variáveis que justificam este fato são encontradas na ausência de infraestrutura tecnológica nas escolas, organização tradicional da distribuição das aulas e professores sem formação ou sem letramento digital. Os estudos são ainda insipientes quando direcionados para o valor dos jogos digitais como relevante estratégia de intencionalidade educativa ao ambiente de aprendizagem [Lourenço 2012], 
V Congresso Brasileiro de Informática na Educação (CBIE 2016)

Anais do XXII Workshop de Informática na Escola (WIE 2016)

[Jappur 2014]. O letramento digital reveste-se de um olhar que o identifica em diferentes espaços de escrita - transpondo a superfície do papel ao meio virtual - os quais geram diferentes modalidades de práticas sociais de leitura e escrita em que o sujeito além de ler e interpretar, tem a possibilidade também de interagir (SOARES, 2002). Significa saber usar socialmente as diferentes mídias disponíveis. Os professores e estudantes utilizam as mídias digitais socialmente, mas tratando-se do espaço escolar, existe uma intencionalidade educativa deste uso que precisa ser considerada nos projetos pedagógicos. A fluência tecnológica se aproxima do conceito de letramento como prática social, e não como simplesmente aprendizagem de um código ou tecnologia; implica a atribuição de significados à informações provenientes de textos construídos com palavras, gráficos, sons e imagens dispostos em um mesmo plano, bem como localizar, selecionar e avaliar criticamente a informação, dominando as regras que regem a prática social da comunicação e empregando-as na leitura do mundo, na escrita da palavra usada na produção e representação de conhecimentos. (ALMEIDA, 2005, p.174).

A partir de tais considerações, alguns dos estudos nacionais [Lima e Soares 2005], [Alves 2005], [Assman 1998, 1996], [Greenfield 1996] analisaram o currículo implícito nos jogos digitais, ou seja, a produção de saberes, habilidades, competências, valores, atitudes e comportamentos que são mediatizados por esses artefatos. Um destes estudos [Lima e Soares 2005] foi realizado com jovens que frequentam ambientes dos jogos digitais (LAN Houses) em cidades brasileiras, uma vez que não há como incorporá-los no ambiente escolar. Os demais estudos estabeleceram amplo diálogo com autores que refletem sobre essa questão [Feuerstein e Falik 2014], [Gee 2004, 2005]. A produção reduzida em relação aos estudos, que analisa os jogos digitais, destaca interligação entre educação, comunicação, interação social e cultural, escolarização e a produção de jogos digitais.

Os jogos digitais são espaços privilegiados de aprendizagem e de ensino por serem inter, intra, multi e transdisciplinares. Eles podem ampliar o desenvolvimento da construção de conhecimentos e habilidades, a interação, a intersubjetividade, o pensamento autônomo e a transcendência elevando o alcance da consciência crítica. Promovem suporte a novos paradigmas epistemológicos da educação, sintonizados com todas as expressões culturais.

Diante de tal contexto, quais conhecimentos e aprendizagens podem ser produzidas pelos jovens? Quais as características específicas dos jogos digitais que podem ajudar com atribuição de sentido e significado para aprendizagem dos conteúdos escolares? Quais soluções podem superar os problemas situacionais como infraestrutura e a burocracia institucional para utilizar os recursos tecnológicos da escola?

Este trabalho, parte de uma tese do IME-USP em desenvolvimento com a FEUSP e tem como objetivo investigar quais os efeitos dos jogos digitais para o ensino dos conteúdos curriculares na escola de Ensino Fundamental II, especificamente na Matemática, com o auxílio das redes sociais (Facebook e WhatsApp) incluindo a perspectiva mobile learning por meio do Bring your own device (BYOD), ou seja, com os dispositivos móveis pertencentes aos alunos. Parte do pressuposto de que os jovens constroem aprendizagens mediatizadas por jogos digitais, desenvolvem inúmeras habilidades cognitivas, afetivas, sociais e culturais e apropriam-se de conhecimentos 
V Congresso Brasileiro de Informática na Educação (CBIE 2016)

Anais do XXII Workshop de Informática na Escola (WIE 2016)

favoráveis à compreensão dos saberes escolares ainda não valorizados pelas famílias e escolas.

A complexidade dos processos de ensino e aprendizagem envolvida no ensino da matemática nas escolas e a ausência de condições favoráveis ao uso de jogos digitais, justificam a relevância de estudos sobre o tema. Se a infraestrutura tecnológica nas escolas públicas não atende minimamente a demanda de alunos e professores, por que não usar os dispositivos móveis, como os celulares?

Na sequência, a seção 2 apresenta a revisão de estudos que contribuem com a fundamentação sobre o tema; a seção 3 descreve o percurso tecnológico de como a pesquisa vem sendo realizada (local, modalidade de ensino, sujeitos, participantes, procedimentos de coleta de dados, equipamentos etc.); a seção 4 apresenta a discussão dos resultados obtidos e, finalmente, apresenta conclusões relacionadas aos objetivos previstos.

\section{Revisão de Estudos}

A produção científica brasileira voltada ao desenvolvimento e aplicação de jogos digitais ligados à educação tem sido reduzida em relação ao desenvolvimento das competências e habilidades existentes no currículo escolar brasileiro.

O recorte temporal definido para revisão bibliográfica teve por base $o$ documento da UNESCO (2013) que contém as diretrizes de utilização de dispositivos móveis em escolas. Tal revisão se ateve aos trabalhos nacionais e internacionais, que levaram em consideração o contexto educacional, cultural e social. De 2013 até 2016 foram verificados na plataforma do Instituto Brasileiro de Informação em Ciência e Tecnologia (IBICT) apenas 17 trabalhos na área de jogos digitais no contexto de ensino e aprendizagem, sendo 13 dissertações e 4 teses.

Lourenço (2012) tratou do "Estado da Arte sobre os Games" a partir do banco de teses e dissertações da CAPES entre os anos de 1987 e 2010. Objetivou mapear e discutir a produção acadêmica brasileira com o tema central ligado aos recursos tecnológicos, em seu estatuto comunicacional, lúdico e educacional. A problematização da pesquisa propôs a discussão sobre a forma em que os jogos digitais são entendidos nas práticas educacionais e o estudo dos meios de comunicação na sua mediação tecnológica com os distintos setores do conhecimento. Muitas das produções analisadas focaram-se sobre questões específicas, tais como a estética, formação de personagens, desenvolvimento de software para games, regulação de comportamentos de gênero, relação dos games com a saúde físico-motora e não uma discussão direta da relação do game com a educação, ou sobre a formação de professores e metodologias que possam auxiliar sobre novos modos de ensinar e de aprender.

O estudo de Jappur (2014) realizou uma pesquisa bibliométrica até 2014 para propor um modelo conceitual de desenvolvimento, avaliação e aplicação de jogos digitais para o contexto do processo de ensino e aprendizagem em sala de aula. Realizou extensiva revisão sistemática em busca de trabalhos nacionais e internacionais relacionadas com a temática.

Câmara (2014) embasado na Teoria de Metas de Realização. Destacou a importância que os jogos digitais representam como estimuladores do desenvolvimento 
V Congresso Brasileiro de Informática na Educação (CBIE 2016)

Anais do XXII Workshop de Informática na Escola (WIE 2016)

dos indivíduos e como meio para motivar o aluno e favorecer a aprendizagem. Investigou a possibilidade da utilização do jogo para dispositivo móvel "Angry Birds" como meio para motivar os alunos pelo processo de ensino-aprendizagem da disciplina de Física. Assim, participaram desta pesquisa 21 alunos do $9^{\circ}$ ano de uma Escola Pública de João Pessoa.

O trabalho de Sena et. al. (2014) apresentou um mapeamento sistemático da literatura sobre aplicativos m-learning utilizados no ensino de matemática com conteúdos e recursos diferenciados, utilizados nos diferentes níveis de ensino (fundamental, médio e superior). Tal mapeamento serviu de base para uma proposta de aplicativo móvel como apoio da aprendizagem de matemática.

A pesquisa de Tabuti e Nakamura (2015) realizou uma revisão sistemática com o propósito de identificar e entender os métodos existentes para o desenvolvimento de jogos digitais de lógica em dispositivos móveis qualificados para a educação. A revisão mostrou que estudos que utilizam ou definem métodos deste tipo ainda não foram explorados na literatura.

Em relação ao uso dos jogos digitais em dispositivos móveis pelos alunos as produções focalizaram seus objetivos nas atividades de interação humano-computador. Estudaram vários tipos de tecnologias processadas por interfaces touchscreens, ondas acústicas, habilidades óticas entre outros recursos. Muitas pesquisas têm avançado em seus designs progressivamente, aperfeiçoando ainda mais os ambientes interativos [Hwang e Su 2011], [Dietz e Leigh 2001], [Wilson 2004], [Brom et. al 2011].

A definição de estudos sobre mobile learning apresentam fundamentação teórica nos estudos de Lave e Wenger (1991) e Pachler (2009) que descreveram que a tradução literal da expressão latina in situ significa na posição, que representa uma exposição sobre a aprendizagem baseada em localização. Afirmaram que "na aprendizagem baseada em localização, os jogadores devem estar em mesma localidade espacial, a fim de compartilhar informações e agir juntos" (p. 721). No entanto, a aprendizagem in situ não poderia articular totalmente a ideia central da contextualização, o que caracteriza a natureza da abordagem mobile lerning. Os pesquisadores afirmaram que "a aprendizagem é como uma atividade situada e concebida do aluno, de suas atividades e do mundo, como mutuamente constitutivas (...). Aqui, a aprendizagem é recíproca, reflexiva e coletiva" (p. 29). Tal postura, conhecida como abordagem de aprendizagem situada, reflete a noção de estudo sobre mobile learning que reitera que o foco deveria estar em contextos, geração e contexto de travessia [Pachler 2009]. Definiram um contexto móvel como "trabalho semiótico e produção de significados que podem ser desenvolvidos pelos alunos, com a ajuda de dispositivos móveis, novas práticas culturais com e através do que eles aprendem. A aprendizagem situada favorece o reforço dos seus recursos no sentido de interagir com o mundo" (p. 5).

Estudo em contexto de aprendizagem móvel de Tan e Hyo-Jeong (2015) destacou que a configuração contextual não pode permanecer constante "como a ação se desenrola onde novos campos semióticos podem ser adicionados, enquanto outros são tratados como irrelevantes" (p. 21). As ações e processos de pensamento experimentam um curso de mudança contínua como novos recursos semióticos do contexto. Como tal, os participantes alteraram seu curso de ações para acomodar, para se adaptar às novas realidades e/ou para seu realinhamento. 
Ainda segundo o estudo de Pachler (2009), da mesma forma e sobre o tema em questão, a natureza fluída e imprevisível de contexto da aprendizagem móvel, reafirma que tal fato "torna-se uma propriedade continuamente e se desdobra nas interações entre as pessoas e seus objetivos, definições e tecnologias. Contexto não pode ser facilmente previsto em termos de avanços pré-determinados, mas pode ocorrer a qualquer minuto pelo celular dos alunos e seus dispositivos" (p. 159).

Outros estudos que teorizaram sobre mobile learning [Orion e Hofstein 1994], [Sharples et. al. 2002] reiteraram que a diferença essencial em relação à aprendizagem móvel de outros tipos de aprendizagem é o fato de que é o aluno que é móvel pois aprende no local onde se encontra; ao invés da tecnologia. Sustentaram preocupação fundamental relativa à compreensão da essência da aprendizagem móvel para compreender como as pessoas se envolvem, com os seus arredores, para criar sites ou redes sociais de improviso para aprender.

\section{Trajetória Metodológica}

O entendimento de que a educação pode ser favorecida por meio do uso de jogos digitais, tanto por ser um fenômeno social quanto como estratégia de aprendizagem, requer pesquisa-ação que possa apreender a realidade em seu contexto. Tal opção atende a pesquisa do fenômeno, de forma ampliada, em relação ao processo de aprendizagem, sem desconsiderar o contexto envolvido por questões sociais, econômicas e culturais. Além do mais, durante o processo de execução da pesquisa o aluno será ou poderá ser melhor influenciado pedagogicamente no processo de ensino e aprendizagem [Barbier 2007].

A Escola Estadual Fernando Nobre localizada em Cotia-SP, campo amostral de desenvolvimento da presente pesquisa, é uma escola estadual de tempo integral. O turno da manhã destinado às aulas "tradicionais" curriculares e o turno da tarde dedicado ao espaço para oficinas, tais como Experiências Matemáticas, Leitura em Língua Portuguesa e Orientação de Estudos, obrigatórias de acordo com a legislação vigente. A escola possui salas de aula amplas, mobiliário em boas condições; uma das salas serve também como sala de vídeo, uma sala pequena destinada à Oficina de Língua Portuguesa e outra sala para informática (15 computadores) além de uma sala para professores.

Por apresentar índices reduzidos na aprendizagem de matemática, no ranking das escolas, por meio de exames externos, esta escola solicitou à professora e pesquisadora Dra. Stela Piconez, especificamente junto ao grupo Alpha de pesquisa da FE-USP, formação tecnológica dos professores para aprender usar as Tecnologias Digitais na sala de aula (Letramento Digital). O Corpo Docente é composto por 22 professores e 220 alunos. Deste total, a Direção da Escola solicitou formação para os professores de Matemática ( $20 \%$ do total) e o uso de jogos digitais para os alunos dos $8^{\circ}$ e $9^{\circ}$ anos da escola. A pesquisa envolveu $20 \%$ de alunos do $8^{\circ}$ ano e mais $20 \%$ de alunos do $9^{\circ}$ ano do Ensino Fundamental II.

Foi aberto um Moodle para os professores como forma de desenvolver competências de Letramento Digital com o compartilhamento de seus projetos com jogos digitais nas Oficinas de Experiências Matemáticas. Para os alunos foram baixados os jogos em seus celulares; o suporte de redes sociais com a abertura de um grupo no 
V Congresso Brasileiro de Informática na Educação (CBIE 2016)

Anais do XXII Workshop de Informática na Escola (WIE 2016)

Whatsapp e outro no Facebook para postagem de dúvidas, compartilhamento dos níveis do jogo e outras interações. Isto se justifica porque a realidade desta escola, em termos de seu projeto pedagógico, plano de aulas, infraestrutura tecnológica reduzida e ausência de formação para uso das tecnologias, foram desafios enfrentados ao longo da trajetória metodológica concebida. De certa forma, contribuíram para adesão à perspectiva do BYOD (Bring Your Own Device) uma vez que há na escola uma sala com apenas 15 computadores e, alguns deles, com defeitos ou desativados, além de burocracia centralizada e necessária para instalação de qualquer aplicativo para atividades docentes. Todos os professores possuem celulares, mas especificamente para uso pessoal e/ou social, como declararam. Diante deste cenário, os celulares foram utilizados sob as perspectivas de BYOD, mobile-learning, Flipped Classroom, blended learning, no contra turno das aulas regulares, ou seja, nas oficinas culturais inseridas no Plano Global da escola.

O BYOD transcende o espaço e tempo permitindo que as atividades se estendam para qualquer espaço. Cada aluno pode ajustar e usar seus dispositivos móveis personalizados no seu próprio tempo, conforto e comodidade. E atende as expectativas que enfatizam as habilidades de autonomia e autoregulação das próprias aprendizagens, fundamentadas pelas Experiências de Aprendizado Mediado (EAM). As EAM não tratam apenas de exposições de estímulos. A presença de um mediador de estímulos ao aprendiz pode provocar interconexões internas e socioculturais como explicitam os estudos desenvolvidos por Vygotsky (2003). Os principais fatores da EAM são tanto o aprendiz, quanto seus colegas e professores portadores de diferentes experiências que tem o papel de exercer influência crucial no desenvolvimento do ser humano. Os mediadores humanos e/ou culturais são responsáveis pelo compartilhamento de elementos culturais mais amplos e mais significativos dos objetos e eventos da experiência direta ou vivenciada pelos aprendizes [Feuerstein e Falik 2014], [Da Ros 2002].

Como procedimentos foram utilizados questionários de sondagem diagnóstica e grupos focais sobre a fluência digital dos alunos. Um conhecimento mais aprofundado do contexto envolveu grupos focais também com os professores no horário de trabalho pedagógico e nas oficinas obrigatórias existentes no currículo escolar. Os alunos selecionaram uma série de jogos; escolheram o "Sim City BuildIt" e foram orientados a jogar durante dois meses, sem qualquer exigência e/ou recomendação pedagógica. No decorrer desse tempo, o pesquisador visitou a escola semanalmente para conversar com os alunos e professores sobre o andamento das atividades, auxiliar em dúvidas técnicas e orientar os professores responsáveis. Os alunos também foram assistidos, acompanhados e motivados através da interação facilitada pelo uso de redes sociais como o WhatsApp e Facebook. Um recurso do jogo permitiu que o mediador pudesse acompanhar o que estava acontecendo a qualquer momento nas cidades dos jogadores. Ao final dos 2 meses 3 pesquisadores do grupo Alpha juntamente com os professores realizaram um grupo focal para a avaliação do que foi aprendido tanto em competências do próprio jogo, como sobre a interação observada com os conteúdos curriculares de Matemática e o entendimento da linguagem abstrata da Matemática.

A trajetória metodológica envolveu diretamente o acompanhamento dos professores no desenvolvimento do letramento digital, da forma como compreendiam a apropriação das tecnologias nas oficinas de Matemática. Ouvindo seus relatos e 
observações para ajustar as atividades ao seu cotidiano, os professores participaram das experiências junto ao pesquisador se adequando e aprendendo a utilizar recursos tecnológicos do m-learning e jogos digitais e, além disso, compreender a necessidade de interação com os conteúdos matemáticos.

Um diário de campo, gravação das discussões nas oficinas sobre as estratégias do jogo e filmagem dos grupos focais também fizeram parte da coleta de dados, de forma continuada, para que se pudesse efetivar a triangulação de dados, com a finalidade de aproximação maior da realidade existente nestas atividades. Os alunos participaram de avaliação coletiva fazendo a transferibilidade dos conceitos matemáticos aprendidos com sentido e significado durante o ato de jogar, para o registro notacional abstrato e formal contido no conteúdo curricular de matemática de seus respectivos anos escolares (Álgebra, Aritmética, Geometria, Geometria Analítica e Porcentagem).

Os resultados obtidos foram fundamentados pela teoria da Modificabilidade Cognitiva Estrutural (MCE) (Feuerstein, 2014) que reconhece o organismo humano como aberto, adaptável e passível de mudança. Diferentes mudanças se dão na estrutura do pensamento de um indivíduo, criando condições para que possa adquirir novas palavras para criar o processo de pensar em novas coisas que previamente não faziam sentido em sua mente. Assume-se que a pessoa é capaz de adquirir de maneira autônoma não apenas diferentes conhecimentos ou habilidades, mas também novas estruturas cognitivas, pelas quais são abertas novas áreas previamente não inclusas em seu conjunto de conhecimentos e habilidades prévias [Feuerstein e Falik 2014], [Da Ros 2002].

Na seção 4 são descritos alguns dos resultados que confirmam as contribuições dos jogos digitais no alinhamento com os conteúdos do componente curricular Matemática nos $8^{\circ}$ e $9^{\circ}$. anos do Ensino Fundamental II.

\section{Resultados}

A pesquisa até a fase onde se encontra foi muito além das expectativas sobre o desenvolvimento de competências previstas, tais como: habilidades de raciocínio lógicomatemático, resolução de problemas, construção de algoritmos adequados e compreensão do registro conceitual e notacional abstrato da matemática com a utilização dos games. Competências não previstas pelo projeto, ou seja, questões sociais envolvendo as responsabilidades cidadãs dos dirigentes e população daquela cidade do jogo SimCity Built surgiram como proposituras dos alunos contemplando a importância do saneamento básico, relevância do planejamento e de escolhas que possam beneficiar os cidadãos. Foram enunciadas questões sobre o fato de que a ausência de construção de escolas eleva os índices de criminalidade e gera necessidade de construção de cadeias. Tais competências foram trabalhadas e discutidas de maneira complexa, analítica e crítica pelos alunos. Estabeleceram relação dialógica trabalhando os conteúdos do jogo de forma multi, inter e transdisciplinar envolvendo outros conhecimentos como os de Economia, Política, Ética, Sustentabilidade do Meio Ambiente etc.

Foi possível observar, todas as competências da Experiência de Aprendizagem Mediada (EAM) revelando a importância do uso dos celulares como estratégia pedagógica na aprendizagem de conteúdos formais da Matemática. Entretanto, os alunos 
identificaram também problemas como ter mais casas para ultrapassagem de níveis no jogo e com isso ganhar mais dinheiro; apontaram para a necessidade de adquirir recursos através da construção das fábricas; ao mesmo tempo, argumentaram que fabricas geram poluição assim como o lixão e as usinas termoelétricas. Foi surpreendente a colocação dos alunos ao mencionarem a importância de ouvir a população que reclama de barulho, poluição e lixo etc.

Durante um dos grupos focais, o pesquisador colocou no quadro da sala de aula três questões envolvendo a resolução de equações do primeiro grau (Álgebra I) contextualizadas com elementos e linguagem presentes no jogo digital. Os alunos resolveram de cabeça a primeira questão, porém foram incentivados a tentar formalizar matematicamente suas respostas usando o elemento incógnita " $\mathrm{X}$ ", nomenclatura desconhecida por todos. Foram estimulados a resolver problemas, a partir do contexto do jogo SimCity Built e de forma colaborativa. Após essa questão, outra mais difícil foi sugerida no quadro, e os alunos após algum momento de reflexão, conseguiram também resolver por meio de equações trocando sugestões e ao mesmo tempo tentando ajudar o colega que estava no quadro. Perceberam que o que estavam tentando resolver era muito semelhante ao que eles pensavam para tomar as decisões no jogo. A partir da segunda questão houve uma comoção dos alunos para tentarem se ajudar mutuamente, cada grupo apresentava uma ideia diferente de como resolver o problema. Ao final, os alunos apresentaram 2 formas diferentes para resolver tais problemas, diante de grande entusiasmo.

A energia, comportamento positivo, motivação e aceitação dos desafios propostos pelo mediador provocou divertimento e satisfação visível nos alunos. Estes, participaram permanentemente de autoregulação das próprias aprendizagens; a autonomia e flexibilidade fizeram parte de todas as atividades e o desempenho alcançado contou com a autoavaliação dos mesmos, o que reforçou o entendimento e compreensão das relações matemáticas, com maior sentido e significado. Os professores, coordenadores pedagógicos e pesquisadores presentes puderam constatar e compartilhar dos apontamentos aqui descritos. Satisfeitos com a experiência propuseram uma nova etapa de continuidade para o uso de outros jogos como por exemplo Minecraft, The Sims e Clash of Clans. Os grupos de Whatsapp e Facebook foram utilizados para: i) tirar dúvidas, que se limitavam à logística do jogo; ii) compartilhamento de informação; iii) motivação; e iv) interação entre alunos e entre aluno e mediador.

\section{Conclusões}

Existem contribuições muito importantes dos jogos digitais para a aprendizagem de conceitos matemáticos. Quando alinhados ao currículo oficial de Matemática, promoveram aprendizagem significativa em relação à compreensão dos registros abstratos deste componente curricular e superam a desmotivação dos alunos. Auxiliaram na melhoria dos escores obtidos nas avaliações externas e nas possibilidades de autoregulação das próprias aprendizagens dos alunos. A composição de dispositivo móvel sob a perspectiva "Bring your own device" (BYOD) com apoio de redes sociais ajudaram na superação dos maiores desafios das escolas públicas: infraestrutura tecnológica precária existente e ausência de formação dos professores. Estes necessitam de formação permanente que os auxilie a utilizar jogos digitais como estratégia 
V Congresso Brasileiro de Informática na Educação (CBIE 2016)

Anais do XXII Workshop de Informática na Escola (WIE 2016)

pedagógica de apoio à construção de conhecimentos matemáticos assim como metodologia específica para uso dos equipamentos móveis na escola com a finalidade de inovar no processo de desenvolvimento e avaliação da aprendizagem dos alunos.

\section{Referências}

Alves, L. (2005). Game over: jogos eletrônicos e violência. São Paulo: Futura.

Almeida, M E B (2005) Letramento Digital e hipertexto contribuições à educação. In: SCHLÜNZEN Jr, K (org.) Inclusão Digital: tecendo redes afetivas/cognitivas. Rio de janeiro: DP\&A

Ardito, C., Lanzilotti, R., Costabile, M. F., e Desolda, G. (2013). Integrating Traditional Learning and Games on Large Displays: An Experimental Study. Educational Technology \& Society, 16(1), 44-56.

Assman, H. (1996). Metáforas novas para reencontra a educação: epistemologia e didática". Piracicaba: Unimed.

Assman, H. (1998). Reencontra a Educação: rumo à uma sociedade aprendente. Petrópolis: Vozes.

Barbier, R. (2007). A pesquisa-ação. Vol. 3. Líber Livro.

Brom, C., Preuss, M. e Klement, D. (2011). Are Educational Computer Micro-Games Engaging and Effective for Knowledge Acquisition at High-Schools? A QuasiExperimental Study. Computers and Education, vol. 57, no. 3, pp. 1971-1988.

Câmara, B. B. A., e Meira. L. R. de L. (2014). Motivação E Games: O Uso Do Jogo Angry Birds Com Estudantes Para O Ensino De Física. Universidade Federal de Pernambuco.

Da Ros, S. Z. (2002). Pedagogia e mediação em Reuven Feuerstein. Plexus.

Dietz, P. e Leigh, D. (2001). Diamond Touch: A Multi-User Touch Technology. Proc. 14th Ann. ACM Symp. User Inter-face Software and Technology, pp. 219-226.

Feuerstein, R.S. e Falik, L. H. (2014). Além da inteligência: aprendizagem mediada e a capacidade de mudança do cérebro. Petrópolis: Vozes Press, pp. 63.

Furió, D., GonzáLez-Gancedo, S., Juan, M. C., Seguí, I., e Rando, N. (2013). Evaluation of Learning Outcomes Using an Educational iPhone Game vs. Traditional Game. Computers and Education, vol. 64, pp. 1-23.

Gee, P.J. (2004). What video games have to teach us about learning and literacy. Palsgrave Macmillan, USA.

Gee, P.J. (2005). Video games, mind, and learning. The Interactive Digital Media \& Arts Association Journal 2.1: 37-42.

Greenfield, P.M. (1996). O desenvolvimento do raciocínio na Era da Eletrônica: os efeitos da TV, computadores e videogames. São Paulo: Summus.

Hwang, W.Y. e Su, J.H (2011). The study of surface computer supported cooperative work and its design, efficiency, and challenges. Interactive Learning Environments, vol.20,no.2, pp.177-198. 
V Congresso Brasileiro de Informática na Educação (CBIE 2016)

Anais do XXII Workshop de Informática na Escola (WIE 2016)

Jappur, R. F. (2014). Modelo conceitual para criação, aplicação e avaliação de jogos educativos digitais (Doctoral dissertation, Universidade Federal de Santa Catarina).

Lave, J. e Wenger, E. (1991). Situated Learning: Legitimate Peripheral Participation. Cambridge, U.K.: Cambridge Univ. Press.

Lima, A. S. J. e Soares, A. (2005). Tecnologias inteligentes e educação: currículo hipertextual. Rio de Janeiro: Quartet.

Lourenço, C. E. (2012). O "Estado da Arte" da produção de teses e dissertações sobre games - entendidos como forma de comunicação - no banco de dados Capes realizadas entre 1987 e 2010. Master's Dissertation, Escola de Comunicações e Artes, University of São Paulo, São Paulo. Retrieved 2016-05-30.

Orion, N. e Hofstein, A. (1994). Factors that influence learning during a scientific field trip in a natural environment. J. Res. Sci. Teaching, vol. 31, pp. 1097-1119.

Pachler, N. (2009). Research methods in mobile and informal learning: Some issues. in G. Vavoula, N. Pachler, and A. Ku-kulska-Hulme (eds.), Researching Mobile Learning: Frame-works, Tools and Research Designs, Peter Lang, Oxford, pp. 1-15.

Roblyer, M.D. (2006) Integrating Educational Technology into Teaching, fourth ed. Merrill Prentice Hall.

Sena, D. M., de Oliveira, E. H. T., e de Carvalho, L. S. (2014). Aplicativos móveis para o aprendizado de matemática. In Anais do Simpósio Brasileiro de Informática na Educação (Vol. 25, No. 1, p. 174).

Sharples, M., Corlett, D. e Westmancott, O. (2002). The design and implementation of a mobile learning resource. Personal Ubiquitous Comput., vol. 6, pp. 220-234.

Soares, M. B. (2002) Novas práticas de leitura e escrita: letramento na cibercultura. Disponível em: http://www.cedes.unicamp.br, Educ. Soc. Campinas, (vol.23, n.81, p.143-160), Campinas

Tabuti, L. M., e Nakamura, R. (2015, October). Métodos para o Desenvolvimento de Jogos Digitais de Lógica: Uma Revisão Sistemática. In Anais do Simpósio Brasileiro de Informática na Educação (Vol. 26, No. 1, p. 41).

Tan, E. e Hyo-Jeong, So. (2015). Rethinking the impact of activity design on a mobile learning trail: the missing dimension of the physical affordances. IEE Transaction on Learning Technologies, vol.8, no. 1, pp. 98-110, USA.

UNESCO (2013). "Diretrizes de políticas para a aprendizagem móvel”. EFA Global Monitoring Report. Organização das Nações Unidas para a Educação.

Vygotsky, L. S. (2003). A formação social da mente: o desenvolvimento dos processos psicológicos superiores. Martins Fontes.

Wang, L. C., e Chen, M. P. (2010). The effects of game strategy and preference-matching on flow experience and programming performance in game-based learning. Innovations in Education and Teaching International, 47(1), 39-52.

Wilson, A.D. (2004). TouchLight: An Imaging Touch Screen and Display for GestureBased Interaction. Proc. Sixth Int'l Conf. Multimodal Interfaces, pp. 69-76. 